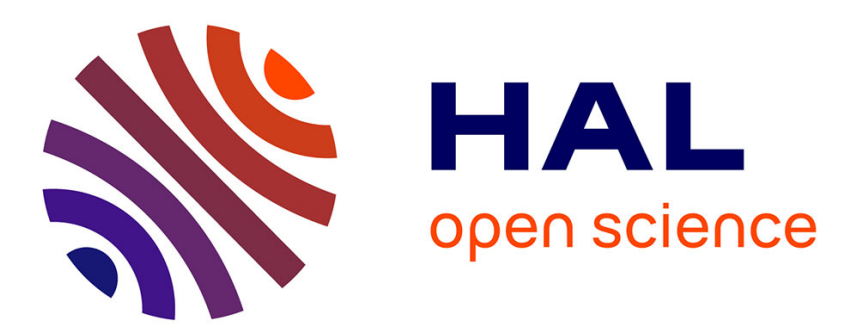

\title{
Rapport 19-05. Contribution au projet d'une loi de programmation pluriannuelle de la recherche
}

Christian Boitard, Bruno Clément, Patrice Debré, Laurent Degos, Didier Houssin, Patrick Netter

\section{- To cite this version:}

Christian Boitard, Bruno Clément, Patrice Debré, Laurent Degos, Didier Houssin, et al.. Rapport 19-05. Contribution au projet d'une loi de programmation pluriannuelle de la recherche. Bulletin de l'Académie Nationale de Médecine, 2019, 203 (6), pp.394-408. 10.1016/j.banm.2019.06.007 . hal02281918

\section{HAL Id: hal-02281918 \\ https://hal-univ-rennes1.archives-ouvertes.fr/hal-02281918}

Submitted on 17 Oct 2019

HAL is a multi-disciplinary open access archive for the deposit and dissemination of scientific research documents, whether they are published or not. The documents may come from teaching and research institutions in France or abroad, or from public or private research centers.
L'archive ouverte pluridisciplinaire HAL, est destinée au dépôt et à la diffusion de documents scientifiques de niveau recherche, publiés ou non, émanant des établissements d'enseignement et de recherche français ou étrangers, des laboratoires publics ou privés. 
Rapport 19-05. Contribution au projet d'une loi de programmation pluriannuelle de la recherche

Contribution to the draft law on multi-annual research programming

C. Boitard ${ }^{1}$ B. Clément ${ }^{2}$ P. Debré ${ }^{3}$ L. Degos ${ }^{3}$ D. Houssin ${ }^{3}$ P. Netter ${ }^{3}$

${ }^{1}$ AP-HP - Hôpital Cochin Broca Hôtel Dieu [Paris]

${ }^{2} \mathrm{NuMeCan}$ - Nutrition, Métabolismes et Cancer

${ }^{3}$ Académie nationale de médecine 
Un rapport exprime une prise de position officielle de 1 'Académie nationale de médecine. L'Académie dans sa séance du mardi 4 juin 2019 , a adopté le texte de ce rapport par 48 voix pour, 17 voix contre et 20 abstentions.

\section{Contribution au projet d'une loi de programmation pluriannuelle de la recherche}

\section{Christian BOITARD (rapporteur), Bruno CLÉMENT, Patrice DEBRÉ, Laurent DEGOS, Didier HOUSSIN,} Patrick NETTER (rapporteur) au nom d'un groupe de travail"

Les auteurs déclarent ne pas avoir de liens d'intérêts avec le sujet abordé.

\section{SYNTHÈSE}

M. le Premier Ministre a annoncé le $1^{\text {er }}$ février 2019 sa décision de lancer le projet d'une future Loi de programmation pluriannuelle de la recherche qui sera déposée au Parlement début 2020. L'Académie Nationale de Médecine a souhaité contribuer aux travaux préparatoires à la rédaction de cette loi en constituant un groupe de travail ad hoc qui a auditionné des personnalités du monde de la recherche (Annexe 1).

Trois évolutions mettent en péril l'avenir de la recherche française: 1- la fragmentation de son organisation et de sa répartition sur le territoire; 2 - le montant et les modalités de son financement, en partie à l'origine de la perte d'attractivité des métiers de la recherche ; 3- l'absence d'une dimension européenne de son pilotage. À chacun de ces écueils, la future Loi de programmation pluriannuelle de la recherche devra répondre par la mise en route du processus d'intégration des différentes dimensions des structures de la recherche, par l'attraction de jeunes chercheurs et enseignants-chercheurs dans un nouvel environnement et par une politique volontariste de recherche concertée en Europe.

1) Pour lutter contre la fragmentation, l'intégration des structures, des moyens et des métiers doit se fixer pour objectif une simplification de l'organisation de la recherche, locale et nationale au bénéfice d'une visibilité et d'une efficience restaurées :

a. Créer une coordination en biologie et santé entre établissements nationaux pour assurer une politique nationale de programmation, d'infrastructures, d'interactions avec le secteur industriel, de relations internationale et confier à cette coordination les moyens exécutifs d'une stratégie commune en la dotant d'une personnalité morale.

\footnotetext{
- Membres du groupe de travail : Jean François ALLILAIRE, Raymond ARDAILLOU, Patrick BERCHE, Bernard BIOULAC, Christian BOITARD (secrétaire), Bernard CHARPENTIER, Claude-Henri CHOUARD, Bruno CLÉMENT (secrétaire), Patrice DEBRÉ, Laurent DEGOS, Antoine DURRLEMAN, Michel HAMON, Didier HOUSSIN, Claude HURIET, Patrick NETTER (président), Bernard NORDLINGER, Pierre-François PLOUIN.
} 
b. Renforcer l'autonomie des universités en modernisant leur gouvernance selon des standards internationaux et favorisant une politique de site intégrant les missions de recherche, de formation et d'innovation, notamment en biologie et santé.

c. Évoluer vers une convergence des statuts des chercheurs et enseignants-chercheurs en définissant selon les cas des valences de recherche, d'enseignement, de valorisation, d'innovation, évolutives en cours de carrière.

d. Simplifier l'évaluation en renforçant son efficacité et mesurant et accroissant son impact.

e. Développer la recherche en Santé Publique et en organisation des soins.

2) Pour rétablir l'attractivité de la recherche dans une concurrence internationale forte :

a. Atteindre la part de $3 \%$ de PIB pour la recherche et le développement, dont $1 \%$ investi durablement dans la recherche publique, et mettre en place un plan d'évolution du financement sur 10 ans.

b. Assurer l'équilibre entre budgets courants des équipes et financements sur projet, avec comme corollaire le refinancement des organismes nationaux de recherche et de l'ANR et redéfinir les sources du financement des sites (préciput, MERRI ...).

c. Revaloriser les rémunérations en prenant en compte les différentes valences auxquelles peuvent prétendre chercheurs et enseignants-chercheurs.

d. Inciter à la recherche partenariale avec l'industrie en favorisant les passerelles avec la sphère socioéconomique et en valorisant cette activité.

e. Favoriser l'innovation: en introduisant dans le jeu l'assurance maladie pour soutenir des essais cliniques indépendants, en introduisant une évaluation des travaux de recherche générés par le crédit impôt-recherche, en favorisant une politique partenariale entre secteurs académique et industriel, en prenant en compte les projets à prise de risque élevée.

f. Définir une politique postdoctorale européenne et internationale en biologie et santé.

3) Pour donner à la France la place qu'elle doit revendiquer dans l'Europe de la recherche

a. Renforcer sa place dans la gouvernance de la recherche européenne en concertation avec la coordination nationale de la recherche pour définir une véritable politique française de l'Europe de la recherche.

b. Corriger la faible représentation des chercheurs et enseignants-chercheurs dans les instances européennes.

c. Avoir une représentation permanente unique de la coordination nationale à Bruxelles pour promouvoir les projets Français en recherche en biologie et santé.

d. Inciter au portage et à la participation d'équipes françaises dans les projets européens.

e. Mettre en place une politique volontariste de recherche concertée en Europe pilotée par un organisme public unique - idéalement la structure de coordination nationale précédemment définie. 


\section{Introduction}

La recherche française dans les domaines de la biologie et de la santé est sous l'autorité de la double tutelle du Ministère des Solidarités et de la Santé et du Ministère de l'Enseignement Supérieur et de la Recherche. Elle a vécu des changements profonds au cours des quinze dernières années. Certains ont été programmés, d'autres sont survenus sans avoir été anticipés.

- L'agence nationale de la recherche (ANR) a été créée en 2004 pour mettre en place un financement de la recherche sur projet.

- Le système universitaire a subi un changement culturel profond avec l'autonomie des universités. Même si celle-ci ne reste qu'une ébauche d'autonomie, elle a conféré aux universités l'obligation d'une réflexion sur la recherche qu'elles hébergent.

- La création des Alliances, dont AVIESAN pour les sciences de la vie et de la santé, pour coordonner l'action d'organismes travaillant dans les mêmes périmètres.

- Les investissements d'avenir ont amené sur les sites des financements selon des modalités nouvelles, mais ont créé des gouvernances parallèles indépendantes des cinq Alliances qui venaient d'être créées, ajoutant à la complexité du système de recherche.

- L'agence d'évaluation de la recherche et de l'enseignement supérieur (AERES), devenu Haut Conseil (HCERES), a été créée en 2006, se substituant au Ministère, aux Établissements Publics à Caractères Scientifique et Technique (EPST) ou à caractère Industriel et Commercial (EPIC) pour assurer une évaluation indépendante ou évaluer des entités qui l'étaient peu ou pas. En a résulté le phasage d'une évaluation tous les cinq ans, introduisant un biais dans les décisions de création des équipes de recherche.

- La Haute Autorité de Santé (HAS) a été créée en 2004 avec dans ses fonctions la certification des Hôpitaux indépendamment de l'HCERES. Des Missions d'enseignement, de recherche, de référence et d'innovation (MERRI) sont apparues en 2005 pour donner un supplément de financement aux CHU. Leur ouverture à tous les établissements de santé a achevé de fragmenter le paysage de la recherche clinique.

Dans ce nouveau paysage, la place des EPST n'a pas été rediscutée. Ils ont gardé un rôle dans la labélisation des équipes de recherche des différents sites. Les programmes hospitaliers de recherche clinique (PHRC) qui avaient été créés en 1992 ont perduré en renforçant leur isolement du reste de la recherche, en particulier biologique.

Durant la même période, les financements se sont dégradés. L'enjeu est l'avenir du pays et sa contribution au progrès des connaissances. Des retombées économiques en sont attendues. C'est le pari que font les pays qui nous entourent.

\section{Le constat}

Le constat sur l'état actuel de la recherche française en biologie et en santé relève des difficultés graves :

- Un financement qui n'a pas évolué en quinze ans alors que le coût des publications a été multiplié par plus de 10, laissant la France au $11^{\mathrm{e}}$ rang mondial en part de PIB consacrée à la recherche et au développement en 2017, derrière l'Allemagne au $7^{\mathrm{e}}$ rang, la Suède, l'Autriche, le Danemark, la Finlande ou la Belgique. En 2017, la part du PIB investie dans la dépense intérieure de recherche et 
développement des administrations était estimée par l'OCDE à $0,77 \%$ en France, $1 \%$ en Allemagne, et dans la dépense intérieure de recherche et développement des entreprises respectivement à 1,42\% et $2,02 \%$. L'Allemagne projette une augmentation du budget alloué à la recherche et au développement de $3 \%$ par an jusqu'en 2030, représentant un total de 1,7 milliards d'euros. Une corrélation est observée dans tous les pays entre les dépenses intérieures de recherche et développement respectives des administrations et des entreprises. Quant à l'efficience des dépenses, le score de performance de la France apparaît avec celui des pays moyennement performants. Le budget des deux grands EPST couvrant les sciences de la vie est voisin. Il était en 2017 de 625,9 millions d'euros à l'INSERM, dont un peu moins des deux tiers dévolus aux rémunérations. La part disponible pour financer les laboratoires labélisés par l'INSERM se situe entre 56 et 57 millions d'euros par an depuis 15 ans. Elle se situe autour de 49 millions d'euros au sein de l'Institut des Sciences Biologiques du CNRS. La part du budget des universités disponible pour la recherche est modeste. Le montant du préciput alloué par l'ANR, en d'autres termes le retour aux établissements hébergeurs, atteint péniblement $11 \%$, soit 55 millions d'euros annuels, un niveau faible en comparaison d'autres pays. Le corollaire en biologie et santé est la difficulté de financer les infrastructures, matérielles et immatérielles, comme les cohortes (Annexe 2).

- La fragmentation des financements. Les financements assurés par l'ANR depuis sa création en 2005 avec un budget d'intervention initial de 669 millions d'euros, ont atteint 834 millions d'euros en 2008 pour se réduire à 527 millions en 2015 et ré-augmenter mais n'atteindre que 708,3 millions en 2019 . Le taux d'acceptation optimal des projets par l'ANR a atteint $18 \%$ avec un financement moyen par projet supérieur à 700000 euros au cours des premières années de fonctionnement de l'agence, pour passer après 2010 à $8 \%$ et remonter à des chiffres de l'ordre de $15 \%$ au détriment du financement moyen par projet, tombé à 450000 euros. Un projet requiert aujourd'hui en France plusieurs sources de financement. Alors que le financement de l'ANR ressort du ministère de la Recherche et de l'Enseignement Supérieur, le financement de la recherche clinique ressort du ministère des Solidarités et de la Santé. Le principal guichet de la recherche clinique est le PHRC dont la dotation était en 2016 de 131 millions d'euros. Ce financement est caractérisé par un cloisonnement étanche qui rompt la continuité de la recherche, de ses aspects les plus fondamentaux à la recherche appliquée, en absence de stratégie assurant une cohérence de l'ANR et du PHRC.

- La fragmentation des structures au fil du temps, avec une visibilité qui s'est encore compliquée en 2009 par la mise en place du « Grand emprunt » rebaptisé « Programme d'Investissements d'Avenir » en mars 2010, dont la gouvernance, remise à un Commissariat Général à l'Investissement, devenu en 2017 Secrétariat Général, n'a pas été coordonnée avec celle des Alliances. Outre les Universités, huit EPST et huit EPIC se partagent la biologie et la santé, avec des périmètres souvent redondants. La multiplicité des tutelles et gouvernances (Annexe 3) est reflétée par le concept d'unité mixte. Bien que les alliances aient été créées pour coordonner l'action d'organismes travaillant dans les mêmes périmètres, l'Alliance nationale pour les sciences de la vie et de la santé (AVIESAN) n'inclut paradoxalement pas tous les organismes ou agences à vocation nationale - l'ANR, chargée de mettre en œuvre la programmation au niveau national, et l'HCERES, chargée de l'évaluation, n'en font pas partie - mais inclut onze organismes associés qui n'ont pas tous une vocation nationale, reflétant la confusion fréquente des niveaux local et national. La Conférence des Présidents d'Université (CPU) et son équivalent hospitalier font partie d'AVIESAN. L'absence de responsabilité officielle d'AVIESAN, autre que celle reposant sur un consensus improbable de ses partenaires, explique que son rôle ait décliné avec le temps. L'absence de personnalité morale limite les ambitions des Alliances au profit de celle des établissements (EPST, ANR). Elle explique le délitement d'une politique de site intégrée qui s'était mise en place dans les premières années d'AVIESAN. En matière d'innovation, les pôles de compétitivité ignorent les Alliances que rien ne lie de manière contractuelle à leur politique. L'absence de lien entre les Alliances et les agences de financement à l'international (AFD, Expertise France entre autres) n'a pas permis une politique incitative et coordonnée à l'international. Le rôle des grandes écoles (Polytechnique, Centrale, ENS...) et du CEA ajoute encore de 
la confusion. Un effet délétère additionnel de la fragmentation des structures, de la multiplicité et du nonalignement sur l'étranger des appellations qui leur sont données explique un défaut de visibilité et lisibilité des laboratoires français à l'étranger et l'incompréhension totale qui en résulte.

- La fragmentation géographique. Si le montant total du financement de la recherche publique, inférieur à celui de nombre de pays européens, est lourd de conséquences, il n'est pas seul en cause dans la dégradation prévisible de la place de la France dans la recherche et le progrès de connaissances. Une dispersion du financement sur un grand nombre de sites est aussi à déplorer. L'évolution de la recherche au cours des 15 dernières années va de pair avec des coûts par projet qui se sont considérablement accrus, nécessitant le recours à des technologies de plus en plus sophistiquées que seules des masses critiques significatives permettent d'atteindre. II est possible que la dispersion géographique et thématique des financements explique le score de performance moyen de la France en comparaison d'autres pays.

- Au-delà de la fragmentation des structures et de la multiplication des tutelles, de l'absence de coordination assumée de l'organisation de la recherche au niveau national, des failles existent dans la programmation de la recherche, comme l'absence de programmes favorisant la transdisciplinarité et la recherche de rupture, l'absence d'une politique nationale concertée à l'international, la prise de risque insuffisante, la tendance à suivre les modes ou encore promouvoir la consanguinité scientifique.

- Les failles dans l'évaluation de la recherche qui, en se complexifiant, en accroissant le nombre de strates successives impliquées, locales et nationales, font de l'évaluation un parcours inefficace, coûteux, conformiste, parfois aléatoire. La complexité de l'évaluation des structures de recherche, de l'unité aux universités ou à l'organisme, reflète la complexité du système. L'inefficacité de l'évaluation est en partie liée au fait qu'elle a en France peu d'impact sur le financement. Le Crédit Impôt-Recherche (CIR), dont l'organisation reproduit celle d'une majorité de pays de l'OCDE, que l'Allemagne applique depuis 2018, est attribué sans évaluation scientifique.

- Des points forts de la recherche biologique et médicale française, qui la place aux premiers rangs mondiaux, sont la recherche sur le cancer, les virus ou les maladies rares, qui s'est inscrite dans le cadre de grands plans de santé publique, très portés politiquement et médiatiquement, souvent en raison d'un secteur associatif très actif. Ce sont les seuls domaines de la biologie et la médecine dans lesquels une visibilité financière a jusqu'à présent été assurée sur le long terme. Ce sont aussi les domaines d'une vision intégrée prenant en compte la programmation, le financement et la mise en œuvre de la recherche, la nécessité de projets multidisciplinaires intégrant jusqu'aux sciences humaines et sociales, l'importance d'une véritable recherche thérapeutique et préventive, d'une politique internationale structurée ou d'une ouverture de la recherche sur la société.

- La perte d'attractivité de la recherche et du métier de chercheur. Elle se traduit par la fuite de chargés de recherche de l'île de France vers les régions, une diminution en 2019 du nombre de candidats au concours de Chargé de Recherche de Classe Normale de $22 \%$ à l'INSERM, dont on redoute qu'elle ne se vérifie dans les années à venir, ou une fuite de chercheurs vers l'étranger. Une raison est le niveau indigne de rémunération des chercheurs (2200 euros nets pour un Chargé de Recherche de Classe Normale) dans une situation internationale concurrentielle. A cette perte d'attractivité s'ajoute une fragmentation statutaire du métier de chercheur qui distingue dans le domaine de la biologie et de la médecine des enseignants, des enseignants-chercheurs, des hospitalo-universitaires, des cliniciens chercheurs ... Les métiers de la recherche biomédicale n'incluent pas en revanche d'ingénieurs dont la fonction est l'innovation technologique, pénalisant celle-ci qui est pourtant une priorité contraignant les laboratoires de recherche publique à faire appel à des prestataires extérieurs privés (disparition des services techniques dans les centres et instituts de recherche, entrainant des surcoûts). L'attractivité est 
enfin mise en défaut par l'inadaptation de l'emploi des jeunes techniciens ou ingénieurs régi par la loi Sauvadet. Les convergences technologiques ouvrent la perspective de nouveaux métiers dans les domaines de l'informatique, de l'intégration de données multiples ou de l'intelligence artificielle, ce qui impose une réflexion nouvelle sur les ressources humaines dans la recherche. La pauvreté en moyens et en investissements structurants de nombreux laboratoires de biologie est un obstacle supplémentaire à l'attractivité. Un corollaire est la faible mobilité des chercheurs. La perte d'attractivité du métier de chercheur va de pair avec la perception par les chercheurs que le temps nécessaire pour la réflexion scientifique et la recherche se réduit du fait de la complexité du système et des contraintes qu'elle génère (charges administratives, dossiers à constituer en réponse aux $\mathbf{N}$ appels à projets pour réunir les fonds nécessaires au travail de recherche, rapports multiples, évaluations ...).

- L'absence réelle de politique de site et de vision régionale. Une grande diversité existe dans les performances des différentes universités médicales et des visions régionales que le nouveau découpage des Régions modifie. Certains Régions ont initié une politique dans le domaine biomédical, tant vis-à-vis des infrastructures que de la mobilité des chercheurs qu'elles peuvent favoriser. Mais pour un site donné, le découplage entre la politique de Recherche Clinique menée par l'hôpital et celle de la recherche menée par l'université et les EPST reste souvent important malgré des tentatives de mise en cohérence par des IHU, RHU, DHU, qui bénéficient à nouveau de multiples dénominations, et la création des CRBSP, qui n'ont qu'un rôle consultatif et n'ont pas prise sur l'utilisation des crédits MERRI créés pour compenser les surcoûts imposés aux CHU par la recherche et l'enseignement. Les MERRI s'élèvent à 1,6 milliards d'euros/an pour l'ensemble des CHU, mais avec un seuil d'admission très bas qui les rend éligibles aux petites structures et aux structures privées, générant un éparpillement de leur distribution.

- La perception d'un déclin de la recherche clinique française. Face aux craintes vis-à-vis des risques portés par l'innovation, au cloisonnement des différents domaines de la recherche en biologie et médecine là où l'interdisciplinarité est pourtant prônée et devrait commencer entre la médecine et la biologie, la place de la médecine française dans le monde est appelée à se réduire. Outre les facteurs d'organisation, d'attractivité, de financement, la rigidité des comités et agences délivrant les autorisations d'études cliniques et l'évolution récente des hôpitaux contribuent à cette évolution.

- La position faible de la France en Europe dans sa représentation dans les instances européennes et dans la course aux fonds. La place de la France dans l'obtention de fonds de recherche dans le domaine de la biologie et de la santé est difficile à évaluer en absence de chiffres autres que globaux agrégeant les mathématiques, la physique ou la biologie et la santé, et les sciences humaines et sociales dont les chiffres par exemple à l'ERC sont pénalisants. La France n'a pas sa place dans le cadre de la recherche européenne en santé au niveau des Directions (DG RTD, DG Santé, DG Connect...) et peu de scientifiques Français sont dans les instances consultatives ou décisionnelles de la communauté européenne. La représentation permanente de la France auprès de l'Union Européenne est à l'image de la fragmentation institutionnelle nationale, sans réelle intégration et action commune en biologie et santé. $\mathrm{Ce}$ vide se retrouve au niveau institutionnel et individuel. Chacun croit avoir les bons réseaux de façon séparée. Si le taux des projets Français acceptés par l'ERC (European Resarch Council) pour la recherche fondamentale biologique demeure acceptable, il est plus faible pour les demandes en recherche appliquée auprès des différentes instances européennes, laissant des pays ayant un accompagnement fortement encadré comme la Hollande ou le Royaume Uni récolter les subventions.

- Au-delà de l'Europe, l'absence de politique internationale coordonnée entre les organismes et institutions de recherche conduit à une fragmentation des représentations diverses de la science française à l'étranger, malgré une initiative heureuse avec la création du programme Montage de Réseaux Scientifiques Européens ou Internationaux (MRSEI) par l'ANR. L'aide au développement fait agir la 
recherche par à-coups au rythme des crises sanitaires (ex. Ebola) tandis que les principaux crédits en santé à l'international, gérés par le ministère de l'Europe et des Affaires Etrangères et dédiés à trois pathologies, vont au multilatéral sans lien avec les initiatives opérationnelles en recherche des EPST ou Universités. Une absence de politique postdoctorale déjà citée pour l'Europe est critique pour l'attractivité des recherches et laboratoires, tant à en Europe que plus généralement à l'international.

- L'innovation tous azimuts impose de nouvelles méthodes d'évaluation et d'organisation des soins. Les produits innovants sont très divers: du médicament chimique aux biothérapies, immunothérapies, thérapies cellulaires et géniques, des dispositifs aux nouvelles imageries, des marqueurs biologiques aux signatures génétiques et épigénétiques. L'irruption du numérique bouleverse la notion de produit avec les applications sur smartphone, la télémédecine, les aides à la prescription, l'alerte aux interactions nocives, l'utilisation des données par des algorithmes de l'intelligence artificielle ou l'analyse de cohortes notamment pour la vigilance. II ne s'agit plus de progrès incrémental ou d'innovation de rupture isolée comme l'ont été les médicaments, mais d'une pluie d'innovations de rupture visant à rendre le soin plus efficace, plus sûr, plus facile et moins invasif ou faciliter les prises en charges ambulatoires. Le mode d'évaluation pour les mises sur le marché est devenu inadapté. Beaucoup d'innovations sont absentes du répertoire des produits de biens et services remboursables. La recherche de nouvelles méthodes d'évaluation (observationnelles, Bayesiennes, retour des bases de données...) et de nouveaux critères de jugements adaptés à chaque type de maladie (Health Technology Assessment research) est nécessaire en complément de celles qui ont promu il y a 60 ans les essais cliniques contrôlés en double aveugle. De nouvelles organisations de soins, flexibles, intégrant des parcours de soins se mettent ainsi en place au gré de décideurs locaux. II n'y a pas en France de recherche sur l'organisation des soins - health service research. Ce champ n'est ni enseigné ni appliqué dans des unités de recherche. L'assurance maladie conduit des expérimentations, mais sans support de recherche.

- Une politique partenariale défaillante avec le secteur industriel. La recherche industrielle a changé : jusqu'en 2005 les industriels cherchaient dans leurs laboratoires des " médicaments vedettes 》 de grande diffusion. Depuis l'affaire du rofecoxib qui a failli mettre en banqueroute Merck, l'industrie s'est reconcentrée sur des "niches " appelées "produits orphelins ", "produits ciblés " puis " médecine personnalisée ". II est difficile de voir des évènements indésirables rares sur une petite population de malades, évitant la formation de recours collectifs à l'origine de procès. Les avantages fiscaux et de concurrence des "produits orphelins", ainsi que l'abandon de la dépense vouée à la communication (publicité dans les journaux, visiteurs médicaux), puisque seuls quelques grands spécialistes concentrent les malades cibles, ont favorisé cette démarche. Les industriels, du fait de la diversité des produits " ciblés », ont arrêté toute recherche fondamentale propre et achètent des start up issues des recherches académiques déjà développées jusqu'aux stades de Phase 1 ou 2. Les transactions ont lieu au plus offrant, à des prix astronomiques. En effet, une recherche tous azimuts pour des " produits de niche " ne peut être entreprise par ces industriels dans le contexte d'une vive concurrence. Cette évolution pose plusieurs questions. Comment redonner l'envie à l'industrie de reprendre une recherche fondamentale pour des produits de large diffusion? Dans le passage entre l'émergence de sociétés venant du milieu académique et le rachat de ces sociétés par des firmes, quelle est la place réservée aux nombreuses instances publiques dans la croissance des start up? PHRC, DRC, incubateurs, pépinières, hôtels, SATT, agences, BPI, où sont coordination, vision, continuité de soutien et même retour sur investissement pour le secteur public ? Comment passer de la fragmentation à l'intégration du développement des produits et de la recherche appliquée ? Et par qui ? Si l'on veut restreindre le choix aux produits qui apportent un bénéfice important, la recherche de l'efficacité d'un produit innovant se juge statistiquement sur peu de patients, alors que l'évaluation de la tolérance exige un nombre considérable de malades soumis au risque (voire l'ensemble des patients). Comment faire évoluer cette recherche ? 
- Une recherche en santé publique et organisation des soins oubliée. Des plans sont favorisés (Haut Conseil pour la Santé Publique, Santé Publique France), mais il y a peu de recherche en santé publique (cf IRDES, EHESP). Pourtant les plans, trop nombreux pour pointer les priorités nationales, devraient être fondés sur les résultats de la recherche. En miroir, il n'existe pas de choix d'indicateurs nationaux (et régionaux) de santé publique, communiqués périodiquement à la population, en dehors du taux de mortalité sur les routes. Une dizaine d'indicateurs nationaux suffiraient pour orienter ces recherches (cf. sécurité routière). La Direction de Recherche des Etudes de l'Evaluation et des Statistiques du Ministère de la Santé (DREES) collige et analyse des données sans faire de recherche primaire. L'épidémiologie pratiquée au sein de l'INSERM est probablement, avec les essais cliniques et des études économiques, la seule recherche fondamentale menée en France. Le GIS-Institut de Recherche en Santé Publique (IRESP), qui a fait suite à l'Institut Virtuel de Recherche en Santé Publique (IVRSP), en étroite collaboration avec AVIESAN, regroupe 28 institutions dont le Ministère des Solidarités et de la Santé, I'INSERM, l'assurance maladie, l'Institut National d'Etudes Démographiques (INED), et soutient des appels à projets sur le fonctionnement du système de santé, les politiques publiques de santé et la prévention, indépendamment de l'ANR. L'IRESP coordonne les actions, communique et valorise les actions de recherche en santé publique avec tous ses partenaires dont chacun veut garder ses prérogatives. Pourtant les priorités ne sont pas affichées, coordonnées, conduites en continuité, des pans entiers de santé publique sont oubliés: l'efficacité de la prévention, l'efficacité des actions contre les addictions, les choix prioritaires de santé publique. Quand considérer un produit ayant un impact en santé publique comme essentiel ? Par exemple, la fabrication de globules rouges artificiels par des industriels étrangers mettrait-t-elle notre pays sous dépendance ? Comment allier veille (Santé Publique France) et vigilance (ANSM, données fournies par les industriels) ? Comment construire des cohortes public-privé pour la recherche en santé publique, le Health Data Hub ne faisant que collecter les données sans en produire? Quelle recherche sur l'autonomie et la dépendance ? Comment construire le partenariat publicprivé en santé publique (pollution, smart cities...) ? Autant d'exemples où les études bibliographiques ou la comparaison avec des pays étrangers, souvent assimilés à de la recherche par nos institutions, ne sauraient suffire.

MAIS, une loi de programmation de la recherche qui : 1- se limiterait à augmenter le financement de la recherche et combler le fossé qui s'est creusé avec les pays environnants sans changer profondément son organisation, 2- ne définirait pas des trajectoires visant, dans le domaine de la biologie et de la santé, à coordonner et intégrer les multiples structures et organismes de recherche sous un guichet unique de coordination nationale, à rétablir l'attractivité de la recherche, développer une politique européenne en biologie et santé,

- en simplifiant notre organisation de la recherche, de ses financements, de ses structures, de leur localisation,

- en maintenant des équilibres fondamentaux entre recherche non finalisée et recherche appliquée ${ }^{2}$, entre financement récurrent et financement sur projet $^{3}$, entre recherche académique et recherche partenariale, entre les sites et un niveau national coordonné, entre une stratégie nationale et une politique européenne et internationale de la recherche.

\footnotetext{
${ }^{2}$ L'idée reçue que le financement de la recherche appliquée est celui qui conduit au dépôt de brevets se heurtent à certains chiffres en particulier les données de l'ERC qui assure pour l'Europe le financement sur projet sans priorités assignées. Les projets de l'ERC génèrent $29 \%$ des brevets obtenus par l'ensemble des projets européens. C'est un chiffre très supérieur à ce qui est attendu si l'on prend en compte la part de l'ERC dans le financement de la recherche européenne, 17\% du financement européen total de la recherche.

${ }^{3}$ Les données de l'OCDE indiquent que l'équilibre entre financement récurrent et financement sur projet relevait en 2019 d'un rapport $75 / 25$ en France, supérieur à celui des pays qui nous entourent. Paradoxalement, pour ce qui est de l'ANR, c'est un rapport inverse, $25 / 75$ qui ressort des chiffres obtenus.
} 
- en rétablissant l'équilibre entre le montant total alloué à la recherche scientifique par la nation et les pays comparables, en particulier européens,

- en réorganisant et décloisonnant la recherche pour accéder à de nouveaux champs de l'innovation, et promouvant les formations à la recherche à l'interface de plusieurs disciplines (médecine et mathématique, par exemple).

exposerait notre pays à une déclin scientifique quî serait, lui, programmé.

\section{QUELLES RECOMMANDATIONS ?}

(1) Aller vers une convergence des statuts des chercheurs et enseignants-chercheurs, prenant en compte le statut principal de chacun selon les grilles de rémunération de la fonction publique, et la répartition du travail en missions définissant des valences qui pourraient être diverses et modulables au long de la carrière : hospitalières comme c'est le cas pour les PU-PH ou MCU-PH, mais aussi translationnelles (les exinterfaces), industrielles, d'enseignement ... Une telle évolution devrait permettre de répondre aussi à l'urgence de la revalorisation de la rémunération des chercheurs et de contribuer à la simplification de l'organisation de la recherche en France qui sont deux aspects essentiels de l'attractivité du métier de chercheur et d'enseignant-chercheur.

Définir des trajectoires pour corriger progressivement la fragmentation des structures, tant locales que nationales. Dans l'attente d'une simplification effective du paysage institutionnel de la recherche en France, des mesures d'urgence devraient être prises pour en décloisonner l'organisation (Annexe 4).

Au niveau national, cet objectif suppose de : 1- utiliser les instances existantes pour introduire une véritable coordination nationale dans les périmètres « régaliens " que sont la politique de site, l'international, les interactions avec le secteur industriel, les infrastructures en biologie et en santé, la programmation; 2confier à cette coordination les moyens exécutifs d'une stratégie commune ; 3 - intégrer dans cette coordination toutes les agences à vocation nationale qui n'y figurent pas, comme les agences de financement et d'évaluation. Cela suppose de subordonner, dans ces périmètres, les décisions des multiples structures et organismes à celle de la structure nationale de coordination; 4- diminuer le nombre de strates d'évaluation de la recherche au niveau national ; 5- regrouper des EPST ou EPIC dont les périmètres se chevauchent largement.

Au niveau local : 1- moderniser la gouvernance des universités en se référant à ce qui est fait dans des pays étrangers, en particulier anglo-saxons ; 2 - renforcer l'autonomie des universités dans l'organisation de la recherche et la labélisation des équipes amenées à soumettre des projets au niveau national ; 3- introduire une vraie coordination de la politique locale de recherche dans les domaines de la biologie et de la santé sous la direction de l'Université. On peut attendre d'une clarification de l'organisation nationale et locale un équilibre que ne saurait assurer la fragmentation géographique à laquelle se heurte la recherche. Il peut seul permettre la création de masses critiques significatives. On peut en attendre aussi une simplification du paysage universitaire permettant une meilleure lisibilité et efficacité régionales. Une politique d'incitation au développement des recherches de rupture et à l'interdisciplinarité est autant de la responsabilité locale que nationale. 
(3) Organiser une vraie recherche en santé publique et en organisation des soins et de la santé (Health service research) grâce à : 1- une politique de formation de haut niveau universitaire (par exemple très peu de Français postulent aux Bourses Harkness du Commonwealth Fund de $130000 \$$ pour un an malgré la communication par l'IRDES) et l'incitation à la création d'unités de recherche en santé publique qui deviennent une urgence nationale ; 2 - la création d'une Délégation à la Recherche et Formation en Santé Publique mêlant formation et unités, ayant une vraie volonté politique, proche du gouvernement et intégrée à la coordination nationale de la recherche, serait une solution comme cela avait été le cas de la DGRST dans les années 1960 pour la recherche biologique.

(4) Favoriser l'innovation : 1- pour répondre aux questions majeures de comparaisons entre produits ou combinaisons de technologies, introduire dans le jeu l'assurance maladie qui pourrait soutenir des essais plus nombreux et ambitieux menés indépendamment de l'industrie, créant par la même occasion un corps d'experts indépendants et compétents. L'expert évaluateur du bénéfice et du risque doit connaître le produit et, pour être indépendant, ne peut acquérir sa compétence que par des essais indépendants (financements publics); 2- introduire une évaluation des projets développés dans le cadre du CIR ; 3- favoriser une politique partenariale entre les secteurs académique et industriel, et assurer la coordination nationale qui peut seule véritablement promouvoir les contacts et collaborations entre les deux secteurs ; 4- établir un lien entre les pôles de compétitivité et la coordination de la politique locale de recherche en biologie et santé ; 5 - revoir les méthodologies d'évaluation de l'efficacité et des risques des nouvelles thérapies d'une part en les dissociant et les dimensionnant à la question posée, et, d'autre part, en considérant l'évaluation en vie réelle appliquant des méthodes observationnelles, des analyses à partir de registres, de cohortes et des bases de données ; 6- favoriser les projets impliquant une prise de risque, en règle écartés par les modes d'évaluation conformistes souvent en place.

(5) Augmenter le financement de la recherche pour 1 - atteindre rapidement $1 \%$ pour la part investie dans la dépense intérieure de recherche et développement des administrations et $3 \%$ pour la part investie de façon globale dans la dépense intérieure de recherche et développement des administrations et des entreprises ; 2- mettre en place un plan d'évolution du financement de la recherche sur 10 ans qui s'inspire du plan allemand; 3- refinancer l'ANR pour lutter contre la multiplication des sources de financement aujourd'hui nécessaires à la mise en œuvre d'un même projet; 4- prendre en compte le financement des infrastructures ; 5- maintenir des équilibres fondamentaux entre la recherche non finalisée et la recherche appliquée, entre le financement sur projet et le financement des équipes par l'augmentation du budget des organismes nationaux et des universités et/ou l'augmentation du préciput défini par l'ANR ; 5- inciter de façon spécifique à la recherche partenariale ; 6- encourager la participation aux appels à projet européens par des mesures incitatives.

(6) Corriger le déclin de la recherche clinique française : 1-favoriser une intégration de la recherche en biologie et santé sur les sites, souvent hospitaliers. Cette trajectoire impose de favoriser sur chaque site un nombre limité d'axes définis comme des priorités locales, l'intégration des activités cliniques et de recherche, intégration que les IHU n'ont pas assuré en raison de leur petit nombre et que les DHU, FHU ou autres ont inégalement assuré en absence de financement significatif, de coordination vraie des parties cliniques et de recherche, de volonté autre que concurrentielle des tutelles; 2- coordonner au niveau national la programmation et le financement de la recherche clinique avec ceux de la recherche translationnelle et fondamentale; 3- aligner sur les standards européens les modalités et l'esprit des décisions des divers comités et agences censés donner des autorisations rapides sur des projets de recherche clinique ; 4favoriser dans la formation des jeunes médecins et biologistes les doubles parcours tels qu'il ont été mis en place par l'INSERM et certaines universités en augmentant leur nombre, et promouvoir les formations multidisciplinaires (par ex: biologie et informatique: IA) les plus à même d'ouvrir sur des ruptures véritablement innovantes en recherche; 5- inciter à la mobilité des médecins universitaires lors de changements de grade ou de fonction comme le font nombre de pays étrangers. 
(7) Une politique volontariste de recherche concertée en Europe avec un seul organisme public idéalement la structure de coordination nationale précédemment définie - qui informe, communique, aide au dépôt de dossiers, gère, et suit les procédures, favorise la présence française dans les différentes instances de la Commission Européenne, et dans les comités d'experts.

En conclusion, il nous paraît essentiel de restaurer la compétitivité de la France dans la recherche et le développement et de faire de la biologie et de la santé des priorités nationales, avec trois orientations: corriger la fragmentation de l'organisation de la recherche, restaurer l'attractivité de la recherche et donner à la France la place qu'elle doit revendiquer en Europe.

Pour atteindre ces objectifs, nous proposons des pistes qui permettront de restaurer la place de la France dans le futur de l'histoire des connaissances:

- restaurer l'attractivité du métier de chercheur en allant vers une convergence des statuts,

- définir des trajectoires qui corrigent la fragmentation des structures tant au niveau national que local,

- introduire une véritable recherche en santé publique et en organisation des soins qui font cruellement défaut,

- favoriser l'innovation qui est un enjeu tant social qu'industriel,

- mettre en place un plan pluriannuel de refinancement de la recherche,

- corriger le déclin de la recherche clinique,

- promouvoir une politique volontariste concertée avec l'Europe.

Les indicateurs qui chiffrent une situation aujourd'hui fragile seront importants pour suivre l'évolution que permettront les mesures que nous avons identifiées (Annexe 5).

Pour copie certifiée conforme

Le Secrétaire perpétuel

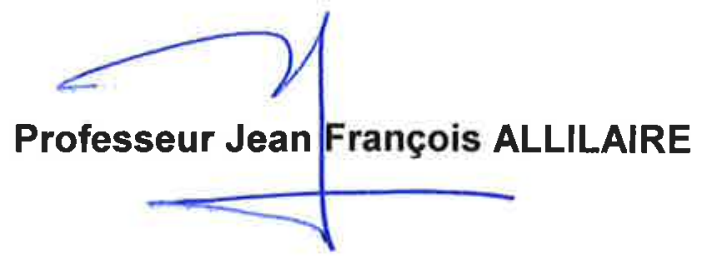




\section{ANNEXE 1. PERSONNALITÉS AUDITIONNÉES ET RÉFÉRENCES}

\section{PERSONNALITÉS AUDITIONNÉES}

Bruno Andreotti, Professeur à l'Université Paris Diderot.

Gilles Bloch, Président-directeur général de I'INSERM.

Alain Bonin, Président de l'université de Bourgogne ;

Jean-Pierre Bourguignon, Président du Conseil européen de la recherche, E.R.C.

Thierry Damerval, Président de l'ANR.

Richard Frackowiak, Professeur, Ecole Polytechnique Fédérale de Lausanne.

Jean-Yves Le Déaut, ancien député, ancien président de l'office parlementaire d'évaluation des choix scientifiques et techniques.

Dominique Dunon-Bluteau, Professeur de I'Université Paris Diderot, responsable du département BiologieSanté de l'ANR.

Pierre Glaudes, Professeur de littérature à l'université Paris-IV , directeur du département de l'évaluation, HCERES.

André Le Bivic, Directeur de l'Institut des sciences biologiques du CNRS.

Ziad Mallat, Directeur de recherche INSERM, Professor of Cardiovascular Medicine, Université de Cambridge.

Isabelle Richard, Conseillère au Ministère de l'Enseignement Supérieur et de la Recherche.

Alain Rousset, Président du Conseil Régional de Nouvelle-Aquitaine.

Jean Sibilia, Président de la conférence des doyens des facultés de médecine.

Pierre Tambourin, ancien directeur général de Genopole.

Nicolas Venteclef, Directeur de recherche à I'INSERM, ERC, Institut des Cordeliers.

Philippe Vigouroux, Directeur du CHU de Bordeaux.

\section{RÉFÉRENCES}

RAPPORT SUR LES POLITIQUES NATIONALES DE RECHERCHE ET DE FORMATIONS SUPÉRIEURES, annexe au PROJET DE LOI DE FINANCES 2018. Document consulté.

France prepares first national research strategy Plan promises funding stability and better career prospects for young scientists. Nature, 2019, 164, 566:164.

German researchers promised a decade of budget increases Government deal adds 1,7 billion through 2030. Science, 2019,364:519.

L'état de l'Enseignement Supérieur et de la Recherche en France, juillet 2018, n¹1. www.enseignementsuprecherche.gouv.fr.

Proposition de la Commission concernant LE PROCHAIN PROGRAMME DE L'UNION EUROPÉENNE POUR LA RECHERCHE ET L'INNOVATION (2021-2027) Horizon Europe. Renzo Tomellini Chef de l'Unité "Politique des programmes HORIZON" renzo.tomellini@ec.europa.eu

L'impact du Crédit d'impôt recherche, Commission nationale d'évaluation des politiques d'innovation. France Stratégie, Gilles de Margerie. Rapport.

Le Déaut Jean- Yves, Sido Bruno, Evaluation de la stratégie nationale de recherche «France-Europe 2020 », OPECST enregistré à la présidence de l'Assemblée Nationale le 6 mars 2017, n45-74 et à la présidence du sénat $n^{\circ} 451$.

Le déclin de la Recherche médicale française Quels constats ? Quelles solutions ? Rapport de la Conférence des Doyens des facultés de Médecine, de la Conférence des Doyens des facultés d'Odontologie, de la Conférence des Doyens des facultés de Pharmacie, de la Conférence nationale des enseignants en maïeutique, 2019. 
La question du financement idéal des laboratoires est complexe. Son enjeu est l'avenir du pays et sa contribution au progrès des connaissances. Les retombées économiques en seront la conséquence, c'est du moins le pari que font les pays qui nous entourent. Le financement de la recherche se pose en termes d'équilibres: 1- l'équilibre entre le montant total alloué aux recherches scientifiques par la nation et les pays comparables, en particulier européens ; 2- l'équilibre entre la recherche d'amont dont l'objectif est d'accroître les connaissances sans a priori sur les avancées qui seront faites, et la recherche finalisée qui vise un progrès dans un domaine biomédical émergent ou fléché ; 3- l'équilibre de la recherche académique et de la recherche partenariale; 4- l'équilibre entre deux modes habituels de financement de la recherche scientifique, le financement récurrent et le financement sur projet. Le financement de la recherche pose aussi les problèmes : 1- de l'évaluation et du mode d'attribution des sommes allouées ; 2 - de la multiplicité des sources de financement qui n'a d'égal que la complexité des tutelles de la recherche française ; 3- du cloisonnement entre la recherche d'amont, la recherche biologique, la recherche translationnelle - un cloisonnement de plus - et la recherche clinique ; 4- l'efficacité du financement de la recherche.

\section{Le montant total du financement.}

Le montant total du financement de la recherche académique, comme celui de la recherche et du développement, est demeuré stable en France depuis 2000. II représente environ $2,25 \%$ du PIB. Mais alors que ce financement était du même ordre en France et en Allemagne en 2000, il a depuis considérablement augmenté en Allemagne comme dans beaucoup de pays environnants.

En 2016, la dépense intérieure de recherche et développement atteignait en France 49,5 milliards d'euros, dont $0,78 \%$ du PIB investis dans la recherche publique (dépense intérieure de recherche et développement des administrations), $1,44 \%$ investis dans la recherche des entreprises (dépense intérieure de recherche et développement des entreprises), soit un total de 2,22\% (sources MESRI-SIES Recherche et INSEE). Le récent rapport parlementaire sur le développement de la recherche, Rapport d'information déposé par la commission des finances en conclusion des travaux de la Mission d'évaluation et de contrôle (MEC) sur l'évaluation du financement public de la recherche dans les universités, enregistré à la Présidence de l'Assemblée nationale le 25 juillet 2018 indique un chiffre de $0,82 \%$ pour la part de PIB investie dans la dépense intérieure de recherche et développement des administrations) en 2017 en 2017. Pour la même année, les chiffres de l'OCDE indiquent $2,19 \%$ en 2017, plaçant la France au $11^{\mathrm{e}}$ rang en part de PIB, devancée en Europe par l'Allemagne qui se situe au $7^{\mathrm{e}}$ rang, la Suède, l'Autriche, le Danemark, la Finlande et la Belgique, mais non le Royaume Uni (16 ${ }^{e}$ rang). E, Israël, ce pourcentage atteint à $4,54 \%$ du PIB. En valeur absolue, la France se situe au $7^{e}$ rang, l'Allemagne au $5^{e}$ rang. La part du PIB investi dans la dépense intérieure de recherche et développement des administrations était estimée par l'OCDE en 2017 à $0,77 \%$ en France, $1 \%$ en Allemagne. Paradoxalement, la Grande Bretagne se place à un rang inférieur à celui de la France, difficilement compréhensible, au regard de sa production scientifique dans le domaine biomédical, si ce n'est par une concentration géographique et thématique des financements.

II existe dans tous les pays analysés une corrélation entre les dépenses intérieures de recherche et développement respectives des administrations et des entreprises. L'une ne semble pas aller sans l'autre. Lorsqu'on regarde l'efficience des dépenses de recherche publique, le score de performance, la France, comme l'Allemagne ou les États-Unis paraissent moyennement performants, derrière la plupart des pays dont la part de PIB dans la dépense intérieure de recherche et développement des entreprises est supérieure à celle de la France, avoisinant le score de performance de l'Autriche, de la République Tchèque ou de l'Italie. Les scores de performance de la Suisse ou du Danemark sont deux fois plus élevés que celui de la France.

En résumé, on peut estimer que le fossé creusé au fil des années entre la France et les pays européens comparables est de l'ordre de $0,2 \%$ pour le secteur public, 0,7 à $1 \%$ pour le secteur des entreprises.

\section{Les sources de financements}

L'ANR est la source de financement quasi-exclusive de la recherche sur projet. L'agence a été initialement créée pour introduire un système de financement sur projet individualisé là où il était antérieurement inclus dans les dotations récurrentes des laboratoires. Là où l'évolution du financement des EPST n'a pas évolué en France depuis les années 2000, le budget de l'ANR a évolué de façon non linéaire depuis sa création en 
2005 avec un budget d'intervention de 669 millions d'euros pour atteindre 834 millions d'euros en 2008,711 millions en 2012 pour atteindre 527 millions en 2015 et ré-augmenter progressivement pour atteindre 708,3 millions en 2019, incluant une réserve de précaution de 54,1 millions d'euros, et 92,8 millions d'euros correspondant au préciput et au financement de l'INCA. Là où l'ANR avait pour objectif initial de surseoir à la fragmentation des financements, imposant pour développer un projet de recherche le recours à des sources de financement multiples. Le taux de succès de l'ANR a atteint lorsqu'il a été optimal $18 \%$ avec financement moyen par projet de l'ordre de 700000 euros au cours des premières années de fonctionnement de l'agence, pour passer après 2010 à $8 \%$ et remonter actuellement à des chiffres de l'ordre de $15 \%$ au détriment du financement moyen par projet qui est tombé à 450000 euros par projet, nonobstant un coût moyen des projets qui a considérablement augmenté pendant la période 2005-2019. La durée des financements sur projet est en règle de 3 ans depuis la création de l'ANR alors que le nombre d'années nécessaires au développement des projets a évolué au cours des années récentes. In fine, bien que l'ANR avait pour objectif initial de surseoir à la fragmentation des financements, force est de constater que pour développer un projet de recherche aujourd'hui, le recours à des sources de financement multiples est une nécessité incontournable. De plus, ce mode de financement n'est enfin pas adapté à la prise de risque qui n'a pas de financement dédié.

Le budget des deux grands EPST dans le domaine des sciences de la vie et de la médecine était en 2017 voisin. II était de 625,9 millions d'euros à l'INSERM, dont un peu plus des deux tiers (64\%) utilisés pour les rémunérations. La part disponible pour les équipes du budget de l'Institut des Sciences de la Vie du CNRS était, lui, de 49 millions d'euros. En lien avec l'augmentation de nombreux coûts, en particulier salariaux, le budget de l'INSERM disponible pour financer les laboratoires n'a pas évolué depuis plus de 15 ans (entre 56 et 57 millions d'euros).

La part du budget des universités disponible pour financer la recherche est modeste. Le montant du préciput alloué par les projets de l'ANR aux hébergeurs, donc aux universités est de $11 \%$, correspondant à un retour aux établissements hébergeurs de 55 millions d'euros. En comparaison, certaines universités américaines ont négocié un préciput qui peut aller jusqu'à $80 \%$. L'enjeu est le financement des infrastructures que ne saurait assurer un préciput de $11 \%$. Le CNRS contribue de façon significative au financement de grandes infrastructures de recherche, par exemple dans le domaine de la physique. La recherche biologique et médicale n'est pas à ce titre sur un schéma analogue, expliquant le coût plus élevé des recherches biologiques à l'ANR, une partie des demandes ayant pour objectifs l'alimenter les besoins relatifs aux coûts des plates-formes technologiques

Le financement par les régions a atteint un peu plus de 1,15 milliards d'euros en 2015, 1,03 milliards d'euros en 2017 d'après les données du SIES. Mais ce financement est caractérisé par sa grande diversité selon les régions et le manque de coordination avec les financements nationaux, en particulier l'ANR. L'importance de l'harmonisation des financements nationaux par l'ANR et des financements par les régions conduit actuellement à une action pilote de l'ANR avec la région Normandie. Une région comme la région Aquitaine Nouvelle a initié une politique en matière de recherche biomédicale, particulièrement dans le financement des infrastructures et des bâtiments et a ouvert des financements sur projets, thématiques ou non et offre des soutiens de mobilité pour les chercheurs seniors et les jeunes chercheurs.

Le financement de la recherche par l'Europe a représenté 7 milliard d'euros sur les 7 ans du programme H2020 (2014-2020) et pourrait passer à 7,7 milliards d'euros sur le programme FP9 (2021-2027). Les projections sur 2021-2027 prévoient 25,8 milliards d'euros sur un premier pilier correspondant au Conseil Européen de la Recherche (ERC)(16,6 Mds€ projetés à comparer à 13 Mds€ sur la période 2014-2020), les Actions Marie Skłodowska-Curie) (6,8 Mds $€$ ) et les Infrastructures de recherche)(2,4 Mds $€$ ), un second pilier correspondant aux problématiques mondiales et compétitivité industrielles, dont $7,7 \mathrm{Mds} €$ consacrés à ce qui correspondait dans le précédent programme à H2020, pour la recherche en santé, mais 15 Mds $€$ dans le numérique et 10 Mds€ dans Alimentation et ressources naturelles en cours de négociation (sortie de la Grande Bretagne et définition de cadre financier pluriannuel ?).

Alors que le financement de l'ANR ressort du ministère de la Recherche et de l'Enseignement Supérieur, le financement de la recherche clinique ressort du Ministère des Solidarités et de la Santé. Le principal guichet en est l'appel d'offres des PHRC dont la dotation était en 2016 de 131 millions d'euros. Ce financement est caractérisé par un cloisonnement étanche qui rompt la continuité souhaitable de l'ensemble de la recherche, de ses aspects les plus fondamentaux à la recherche appliquée. A titre de référence, les dépenses de santé remboursées par la Sécurité sociale s'élevaient la même année à un peu plus de 150 milliards d'euros, 
pratiquement $78 \%$ de la consommation de soins et de biens médicaux ( 200 milliards, $8,7 \%$ du PIB, un peu plus de 2900 euros par habitant), indiquant un investissement pour le moins limité. Le financement en santé pose le problème de sa visibilité : côté État, il n'existe pas de programme généraliste de recherche en santé, comme il en existe dans d'autres domaines comme la défense, l'agriculture, l'environnement... Côté Assurance Maladie, les financements de la recherche clinique de type MERRI ou PHRC sont mal assumés et peu visibles. La recherche biomédicale est dans une période de déclin. Les causes sont systémiques : complexité de son organisation, carences des interactions entre les acteurs, déficit/complexité des financements, perte d'attractivité notamment pour les médecins, compétition internationale. II y a un grave déficit de financement des emplois en recherche médicale. On observe $+21 \%$ de recrutement des $\mathrm{PH}$ dans les $\mathrm{CHU}$, mais pas de progression de recrutement de HU. L'investissement dans le domaine de la santé publique a enfin été historiquement défaillant.

La Fondation de la Recherche Médicale, qui est en France le financeur caritatif le plus important, touchant tous les domaines de la recherche biomédicale, a financé la recherche à hauteur de 43,6 millions d'euros. La fondation ARC alloue 25 millions d'euros par an, la ligue contre le cancer 38,9 millions d'euros en 2016. Le budget de Recherche sur le Sida était la même année d'un peu plus de 50 millions d'euros.

Le crédit d'impôt recherche (CIR) est, avec une dépense fiscale d'environ 6 milliards d'euros/an, la $2^{\mathrm{e}}$ niche fiscale après le CICE. Créé en 1983, modifié en 2004 et surtout 2008, il incite depuis 2008 aux partenariats avec les laboratoires académiques et à l'emploi de jeunes docteurs. II représente environ $60 \%$ de l'ensemble des aides publiques à l'innovation. En 2017, 30 des 35 pays de l'OCDE étaient pourvus de dispositifs fiscaux en faveur de la R \& D. L'Allemagne a décidé en février 2018 de réintroduire chez elle ce type de dispositif fiscal, au moins en faveur des PME (cf. Rapport de la Commission nationale d'évaluation des politiques d'innovation présidée par Gilles de Margerie). Seul 20\% des quelques 6 milliards auxquels correspond le CIR vont aux PME. Le CIR pose un problème propre d'évaluation scientifique de ce qui est vraiment de la recherche dans ce qu'il finance, ce qui reste à évaluer. Le rapport indique que les entreprises qui bénéficiaient du CIR avant la réforme de 2008 ont augmenté leurs dépenses de R \& D d'un montant égal ou un peu supérieur à celui de l'aide fiscale reçue, que le nombre d'entreprises déclarantes a été multiplié par 2,6 depuis 2008 , avec un impact moindre sur l'emploi de personnels de $R$ \& D que sur les dépenses de R \& $D$, mais néanmoins une réduction du temps d'accès des docteurs aux emplois de $R \& D$ avec effet de substitution des ingénieurs par des docteurs de spécialités " ingénieurs ". Un euro additionnel d'aide publique allouée via le CIR induit un euro de dépenses supplémentaires de $R \& D$, un bilan comparable à d'autres pays de l'OCDE. Des effets positifs souvent faibles ont été rapportés sur la probabilité d'introduire des produits nouveaux, sur le dépôt de brevets, sur la productivité, sur l'attractivité internationale (cf. Rapport de la Commission nationale d'évaluation des politiques d'innovation présidée par Gilles de Margerie).

\section{L'équilibre des financements.}

II n'existe pas de chiffre connu indiquant l'équilibre entre la recherche publique non finalisée, sur laquelle reposent initialement beaucoup d'avancées médicales, et la recherche publique appliquée destinée à appuyer le développement de domaines émergents et d'applications médicales. II est probable que cet équilibre dans le domaine biomédical est voisin de $50 \%$. Au niveau européen, la part de l'ERC était jusqu'à présent mineure (14\%), Horizon Europe comptant pour $86 \%$ dans cet équilibre. Le prochain programme cadre prévoit une augmentation du financement de l'ERC. Dans le budget de l'ANR qui va au financement des projets, près de $75 \%$ vont aux projets génériques, non fléchés.

Plus globalement, les données de l'OCDE indiquent que l'équilibre entre financement récurrent et financement sur projet relevait en 2019 d'un rapport 75/25, inverse de celui indiqué par l'ANR, supérieur à celui des pays environnants : 36/100 en Allemagne, 53/100 en Grande-Bretagne, 28/100 en Suisse. Un handicap du financement par projet en France a été indiqué avec l'obligation de recourir à des sources de financement multiples pour un même projet. La même observation tient d'ailleurs pour le financement récurrent des laboratoires qui est soumis à la multiplicité des tutelles. Le financement des équipements requiert souvent lui aussi de multiples partenaires : les EPST (parfois plusieurs), l'Université, la Région.

Si le montant total du financement de la recherche publique inférieur à celui de pays européens environnant est lourd de conséquences, il n'est pas seul en cause dans la dégradation attendue de la place de la France dans la recherche des connaissances. Une dispersion du financement sur un grand nombre de sites pourrait être une partie de l'explication. L'évolution de la recherche au cours des 15 dernières années va de pair avec des coûts par projet ou par publication qui se sont considérablement accrus, nécessitant le recours à des 
technologies de plus en plus nombreuses et sophistiquées que seules des masses critiques significatives peuvent atteindre. Concentrer les financements est avancé par certains pour expliquer la position paradoxale de la recherche en Grande-Bretagne.

En conclusion, l'ensemble des chiffres précédents conduit à la proposition de corriger le déficit de l'investissement public dans la recherche et le développement et de restaurer l'équilibre avec les pays européens qui se projettent dans l'avenir. L'Allemagne dont la part d'investissement public dans la recherche et le développement atteint $3 \%$ du $\mathrm{PIB}$, correspondant à un budget recherche de $33 \%$ supérieur à celui de la France, projette dans le cadre d'un plan pluriannuel une augmentation supplémentaire de $3 \%$ par an jusqu'en 2030, un total de 1,7 milliards d'euros. L'OPECST dans son rapport de 2017 sur l'évaluation de la stratégie nationale de recherche préconisait une programmation pluriannuelle et une augmentation annuelle pendant 5 années consécutives de 700 à 900 millions d'euros pour la recherche, dont $40 \%$ des crédits seraient consacrés au secteur biologie-santé, soit une augmentation budgétaire de 280 à 360 millions d'euros par an. Celle-ci devrait permettre la sanctuarisation des efforts de recherche fondamentale, la revalorisation des carrières et le financement d'un dispositif de résorption de la précarité de l'emploi scientifique dû à la loi Sauvadet. Cet organisme parlementaire indiquait que la meilleure façon de hisser la biologie santé au rang de priorité nationale serait de consacrer une action prioritaire à la convergence des NBIC (nano-bio-info-cogno) et d'impliquer les Sciences Humaines et Sociales dans les grands défis. 


\section{Annexe 3. Complexité du paysage français de la recherche}
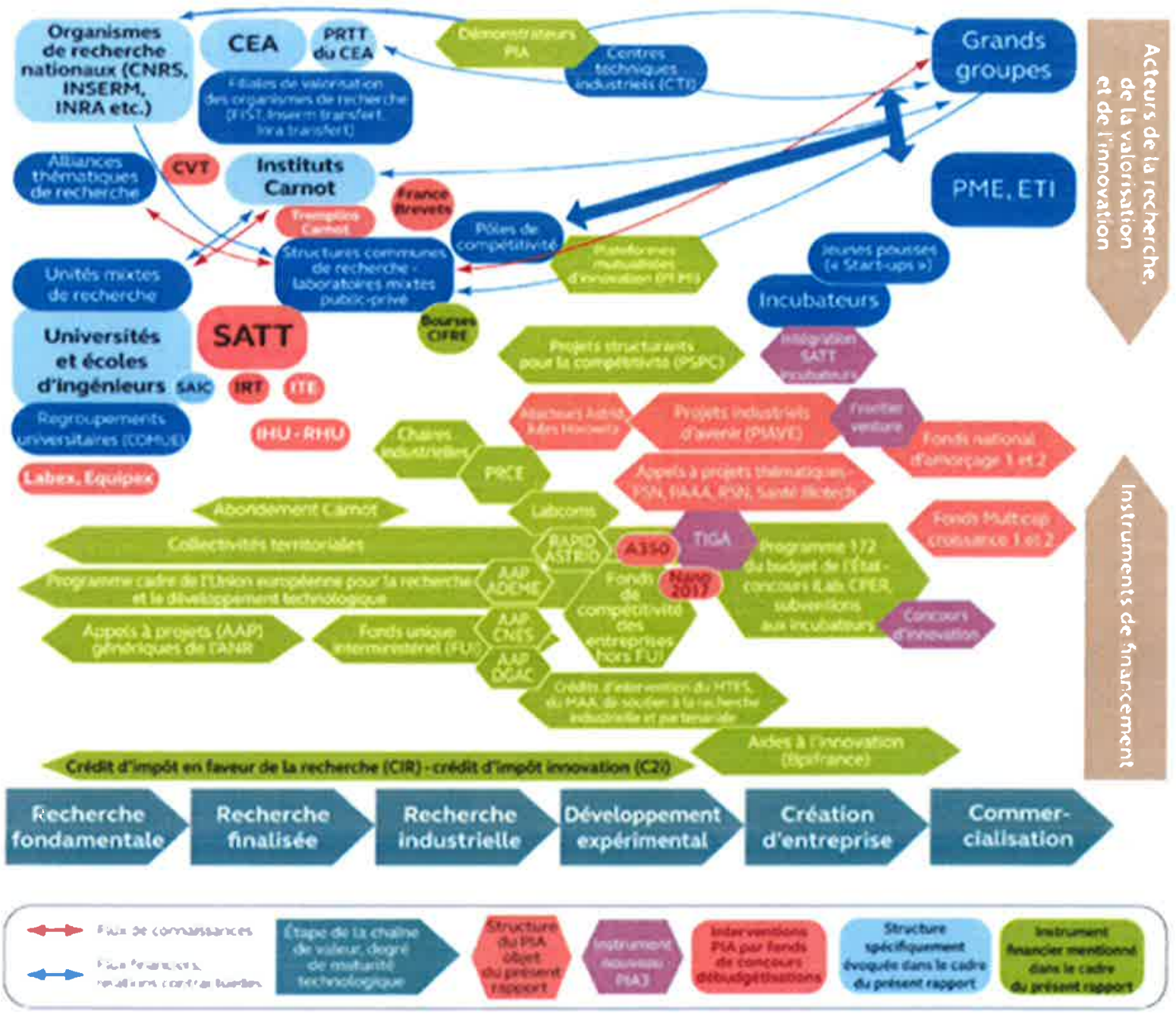


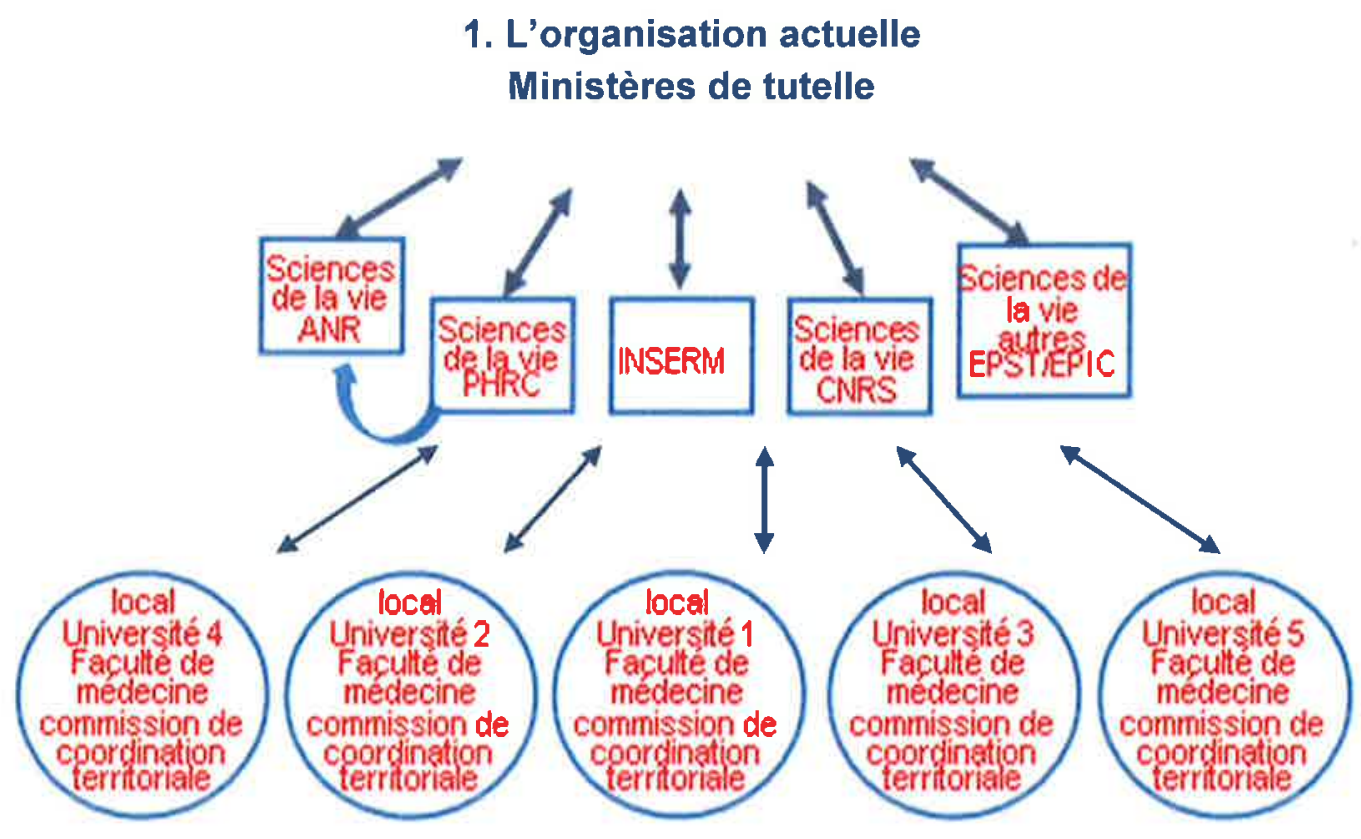

L'organisation actuelle (1) est caractérisée est caractérisée par : 1- la multiplicité des structures nationales dont la coordination sous l'égide d'alliances, AVIESAN dans le cas de la biologie et la santé, fonctionne de façon chaotique en raison de l'absence de périmètre de compétence clairement défini ; 2- un grand nombre d'universités au niveau local, une réforme en 2007 qui n'a conféré aux universités qu'une ébauche d'autonomie ; 3 - des structures nationales, en particulier les EPST, dont la place n'a pas été redéfinie en regard de cette autonomie ; 4- une fréquente confusion des niveaux local et national, par exemple dans la labélisation des équipes qui est restée nationale tout en étant influencée de façon variable par le niveau local.

Une organisation simplifiée doit impérativement : 1- redéfinir le niveau national en le simplifiant (regroupement d'EPST ou EPIC dont les périmètres sont redondants), en lui conférant une coordination effective et les moyens exécutifs d'une stratégie commune (2a) avec des périmètres définis (l'international, la société, les financements sur projets, les infrastructures, une politique postdoctorale) dans le domaine de la biologie et de la médecine sous l'égide de ses ministères de tutelle (2b); 2- impliquer un niveau local renforcé, lui permettant d'assurer ses missions régionales, par exemple ses interactions avec les Régions ou, dans le champ de l'innovation, avec les pôles de compétitivité (2b). 
2a. Une organisation simplifiée Une coordination nationale

Un niveau local et un niveau national distincts

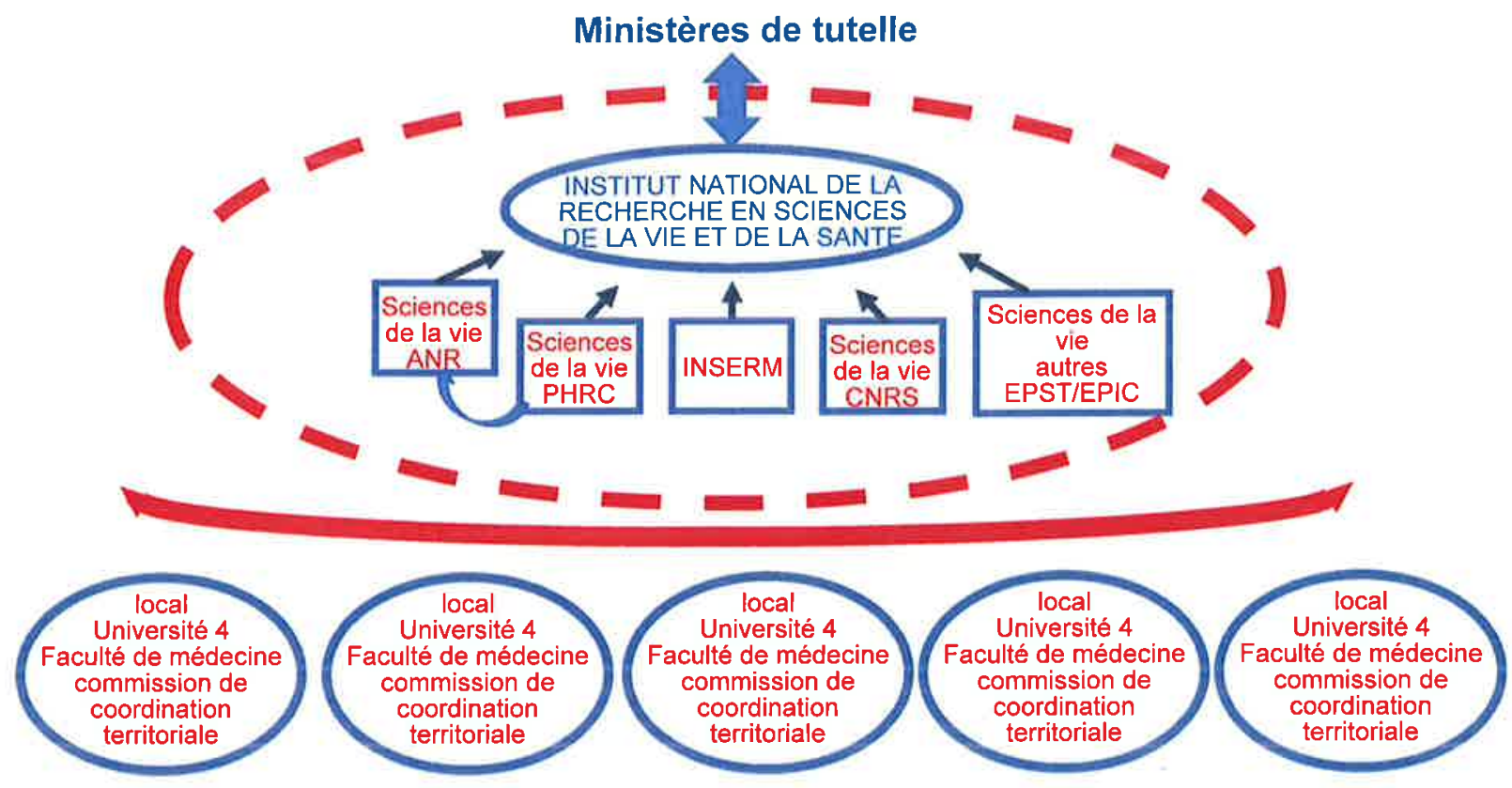

2b. Une organisation simplifiée,

des périmètres de compétence effective définis :

Coordination : l'international, la société, les financements sur projets, les infrastructures, une politique postdoctorale.

Sites : politique de site, liens avec la Région, politique régionale d'innovation

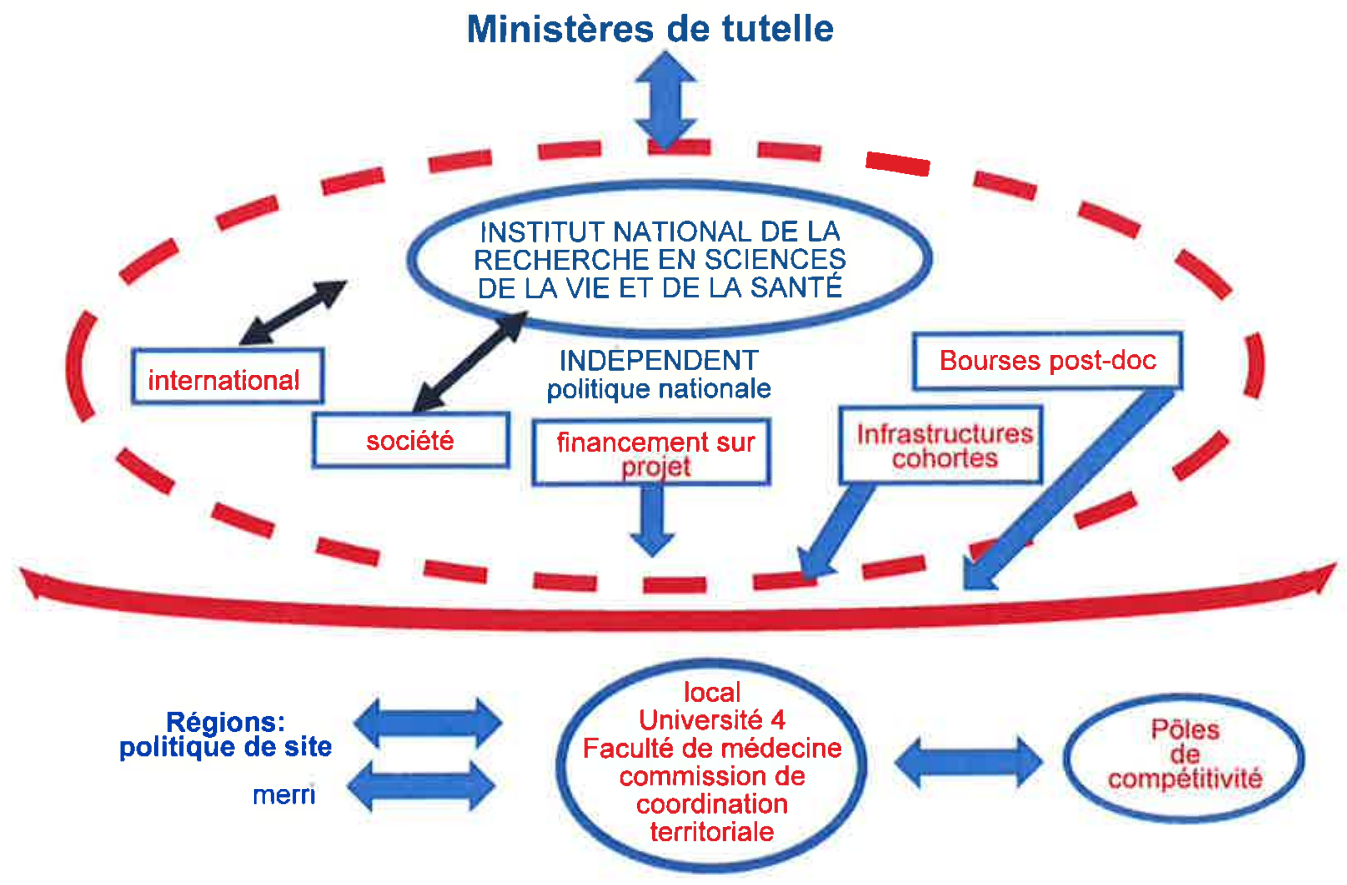

UNIVERSITÉS AUTONOMES politique de site 


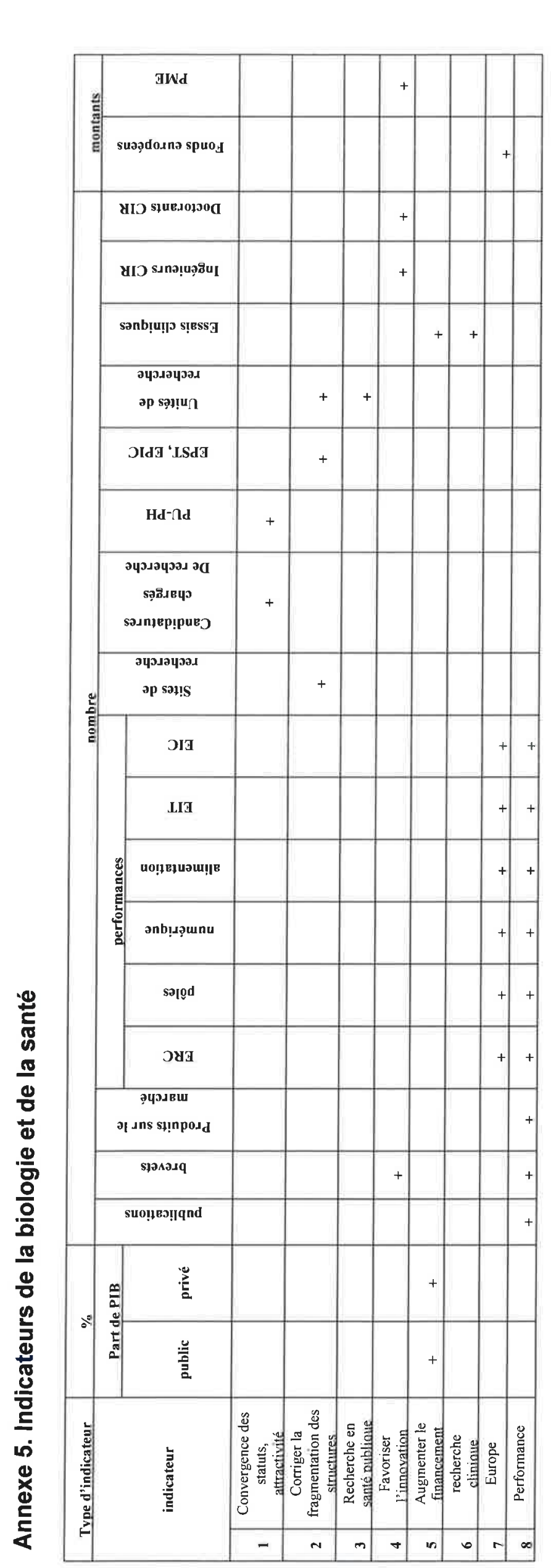

ธิธี

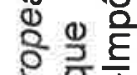

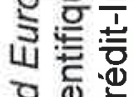

ฮ. $\frac{1}{0}$

赵

$\stackrel{\square}{\square}$

स. क

บ

Ш

- 0

ठ․

유

헝 음 음

은

ठ क्ष

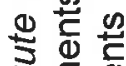

농

乌

조음

응.4

岗ら

ビ岀品

एँ

흥 $\frac{\bar{\alpha}}{\overline{0}}$

亏웡

잉요잉

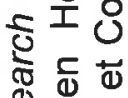

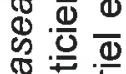

野

눙

ญิ.

อั कू

Uు 웡

ن

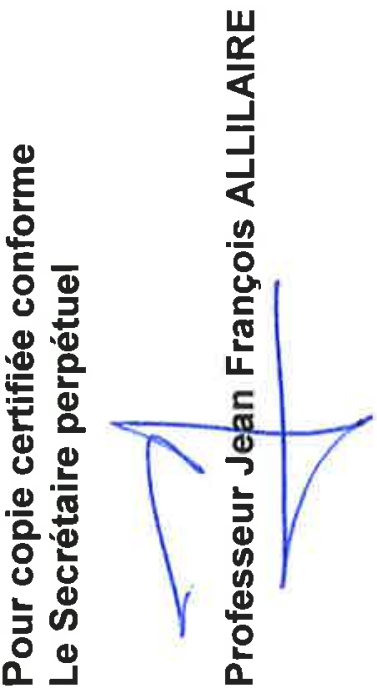

品娄

…일

공호응

인ㅇㅁㅇ

远造点

产은 它 잉

드 통

芌寅总

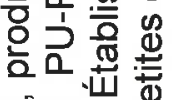

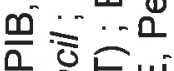

‥ 을

ㄷํㅇ몀

을

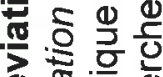

ब

응 응 덩 